\section{Estudo da clientela do Programa de Atendimento Odontológico Precoce em um serviço público do município de Cuiabá, Mato Grosso}

\section{Study Program of Early Dental Care clientele of a public service in the city of Cuiabá, Mato Grosso, Brazil}

Luiz Evaristo Ricci Volpato 1

Ângelo Falcão de Figueiredo 2

1 Faculdade de Odontologia. Universidade de Cuiabá. Av. Beira Rio, ${ }^{\circ} 3100$, Jardim Europa. Cuiabá, MT, Brasil. CEP: 78.015-480

2 Instituto de Saúde Coletiva.

Universidade Federal de Mato Grosso. Cuiabá, MT, Brasil.

\begin{abstract}
Objectives: to describe the children assisted by a public clinic Program of Early Dental Care in Cuiabá, identifying: a) the age group of the children when entering the program; b) the use of the bottle in each age group; c) the use of the bottle at night; d) the accomplishment of oral hygiene; and e) the presence of dental caries.

Methods: a cross-sectional study was performed using 934 medical files of the children in the program between 1996 and 1998.

Results: $92.93 \%$ of the children entered the program aged from 0 to 36 months; until the $42^{\text {nd }}$ month of age, in only one age group the number of children that used the bottle was lower than the number of children who did not; a large number of the children used the bottle at night; both oral hygiene and dental caries prevalence were directly associated to the children's age.

Conclusions: it is necessary to raise population awareness of the value of oral health as the program indicated a large number of children who were weaned much too early and only one third of the children in the program had any oral hygiene at all during the first year of life.
\end{abstract}

Key words Public health, Community dentistry, Public health dentistry, Pediatric dentistry

\section{Resumo}

Objetivos: descrever a clientela assistida pelo Programa de Atendimento Odontológico Precoce de uma clínica municipal de Cuiabá, identificando: a) faixa etária da clientela ao iniciar-se no programa; b) uso de mamadeira por faixa etária; c) uso da mamadeira noturna; d) realização de higiene bucal; $e$ e) presença de cárie dental.

Métodos: estudo descritivo de corte transversal, utilizando os 934 prontuários das crianças atendidas pelo programa entre 1996 e 1998.

Resultados: 92,93\% da clientela do programa era composta por crianças que iniciaram o tratamento com idade entre 0 e 36 meses; até os 42 meses de idade, em apenas uma faixa etária o número de crianças que usavam mamadeira é inferior ao número de crianças que não faziam uso da mesma; entre as crianças que usamvam mamadeira, a maioria absoluta o fazia durante a noite; a realização de higiene bucal e a prevalência da cárie dentária foram diretamente relacionadas à idade da criança.

Conclusões: é necessário um trabalho de conscientização da população quanto à saúde bucal visto que a clientela do serviço estudado apresentou alto indice de desmame precoce e a higiene bucal esteve presente no primeiro ano de vida em apenas um terço das crianças assistidas.

Palavras-chave Saúde pública, Odontologia comunitária, Odontologia em saúde pública, Odontopediatria 


\section{Introdução}

Em 1948, a Organização Mundial de Saúde (OMS) conceituou Saúde como o bem estar bio-psíquico social dos indivíduos. Desde então, essa concepção tem sido discutida e mais recentemente criticada. Durante a VIII Conferência Nacional de Saúde, realizada em 1986, em Brasília, buscou-se criar parâmetros mais concretos para definir saúde, resultando em uma conceituação na qual as condições de saúde passaram a ser relacionadas a condições de trabalho, alimentação, moradia e lazer, entre outras. 1

Nessa dimensão, ao lado da higidez física, características como liberdade interna, autodeterminação e capacidade de adaptação e integração ativa e criativa no universo social são também atributos de saúde.

Assim sendo, a saúde é considerada não só uma condição individual, mas populacional, bem como a não tão somente ausência de doença, mas a mobilização dos indivíduos na busca da satisfação das suas necessidades, características da cidadania. A saúde não é algo que se tem ou que se recebe como doação ou resultado de assistência - seja médica, odontológica, psicológica ou outra qualquer -, mas sim um estado de consciência e de condições de vida material e social que possibilitam o crescimento e a realização plena das potencialidades de cada indivíduo, em outras palavras, se trata também de questões de cidadania. ${ }^{2}$

Essa visão de saúde é fruto recente de estudos e de conferências mundiais abordando o tema e que ainda não está presente na consciência da população. Encontra-se aí inclusa grande parte dos profissionais de saúde que não estão em consonância com as discussões atuais sobre a questão. Mesmo os profissionais que atuam diariamente no serviço público encontram dificuldade em modificar a sua ação e adotar uma postura de promotor de saúde. Tal dificuldade para abordar holisticamente o paciente devese também às universidades e entidades de ensino superior que ainda pregam a visão fragmentada do indivíduo, enxergando-o em partes; criando cada vez mais especialistas que não conseguem visualizar o indivíduo como um todo, apenas suas partes distintas. O aprofundamento dos especializados está em consonância com o conceito anterior de ausência de doença; o conceito atual procura sempre priorizar o indivíduo integral. O profissional de saúde e o odontólogo não fogem à regra; ainda são vistos como alguém que irá solucionar um problema que o indivíduo apresenta quando na verdade eles deveriam empregar seus esforços no intuito de evitar que esse problema se instale.

A Odontologia, tradicionalmente, vem colocando seus pacientes numa condição passiva em relação aos problemas bucais que os afetam, sendo aceita como natural por ambas as partes envolvidas. Os procedimentos curativos ou de limitação de dano exigem muito dinheiro e pouco envolvimento do paciente com o tratamento. A postura curativista do odontólogo, agindo após a instalação da doença bucal no sentido de combatê-la e assim restabelecer a saúde, está inserida no que Berlinger ${ }^{3}$ chamou de relação causa e efeito. $\mathrm{Na}$ relação causa e efeito encontra-se a base empírica do antigo conceito de saúde - ausência de doença - não sendo mais aceitável nos parâmetros da saúde atuais.

Uma das maneiras mais importantes para mudar a mentalidade curativa-restauradora arraigada na população, gerando a prevenção, é através da educação. A educação é um instrumento de transformação social, não só a educação formal, mas toda ação educativa que propicie a reformulação de hábitos, aceitação de novos valores e que estimule a criatividade, 2 aspectos esses da educação em saúde.

A cada dia mais se acumulam evidências da necessidade de cooperação do paciente e da importância de mudança de comportamento em relação à saúde bucal. Para tanto, sem desprezar as questões socioeconômicas, o cidadão é estimulado a refletir sobre o seu papel como responsável pela sua saúde bucal e daqueles que educa, segundo Rossetini e Moreira, citados por Corrêa. ${ }^{2}$ Isso está de acordo com a nova conceituação de saúde, que procura uma relação mais dinâmica do indivíduo em busca do seu bem-estar.

A saúde bucal, assim como a geral, deve ser buscada de maneira preventiva; devemos lançar mão de instrumentos ou métodos que nos auxiliem a evitar que se instale a doença, no caso, a cárie dentária. A maneira de se evitar a instalação da cárie é através de programas preventivos para as crianças, pois "crianças sadias tornar-se-ão adultos sadios". E quanto mais cedo essa ação preventiva tiver início melhor será o resultado alcançado. 4

A assistência odontológica a crianças menores de seis anos sempre se constituiu num problema no Brasil em virtude da prática consolidada de se manter como grupo prioritário os escolares de 6 a 14 anos. $4-6$

Sob o lema "educar prevenindo e prevenir educando" e atendendo a uma tendência mundial de atenção odontológica em crianças de baixa idade, com o estabelecimento de programas educativos e preventivos para o controle da cárie dentária, surgiu em 1985 a "Odontologia para Bebês na Universidade 
Estadual de Londrina".7

Contrariando um consenso dentro da Odontopediatria de que a época ideal para iniciar os exames e tratamentos dentários era por volta de dois a três anos de idade, a nova filosofia propunha o atendimento odontológico à criança antes de completar o primeiro ano de vida (podendo iniciar com a gestante), para realizar manobras preventivas no âmbito doméstico, como a limpeza da boca, o controle da amamentação noturna após os seis meses, o consumo "inteligente" dos açúcares e a aplicação tópica de flúor.7

Estudos realizados por Walter et al. 4 em 1987 , demonstraram que a prevalência de cárie em crianças de 0 a 30 meses era alta (34\%), e que observava uma característica muito peculiar, que condizia com dados mundiais: o aumento da prevalência de cárie entre o primeiro e o segundo ano de vida. Portanto, grande parte da população menor de três anos, que não eram beneficiadas pela assistência odontológica tradicional, já apresentavam necessidades curativas antes mesmo do primeiro exame de rotina. Concluiu-se que o atendimento odontológico deveria iniciar antes da possibilidade do aparecimento da doença, ou seja, no primeiro ano de vida. 4,7 Além dos resultados significativos na redução da prevalência e severidade da cárie, um dos grandes méritos do programa de odontologia para bebês foi ter introduzido uma nova mentalidade nos profissionais e nos usuários, na medida em que estes passaram a procurar o serviço para atividades preventivas com uma maior freqüência. 5

Nakagawa, ${ }^{8}$ em 1995, apresentou projeto para a incorporação do programa de atendimento odontológico no primeiro ano de vida no Município de Cuiabá. A estratégia da "Bebê-Clínica", no entanto, foi agregada aos demais serviços de assistência odontológica do Município de Cuiabá em 1996 com a implantação da primeira clínica odontológica do município, a "Clínica Odontológica do Verdão". 9 As clínicas odontológicas que foram inauguradas posteriormente também vieram a contar com o serviço de atenção odontológica precoce (Clínica Odontológica do CPA III, do Jardim Leblon, do Coxipó, do Dom Aquino e do Pascoal Ramos, além do Centro de Saúde Escola do Grande Terceiro).

É essencial a realização de estudo sobre o programa para que esse possa ser avaliado e com isso gerar subsídios para a sua implantação em diferentes municípios e, havendo a necessidade, sua posterior modificação.

O objetivo do presente estudo é descrever a clientela assistida pelo programa de atendimento odontológico precoce para crianças de 0 a 5 anos de uma clínica odontológica do município de Cuiabá.
Pretende-se assim: a) identificar a faixa etária da clientela atendida no Programa de Atendimento Odontológico Precoce para crianças de 0 a 5 anos, ao iniciar-se no programa; b) verificar o uso de mamadeira pelas crianças atendidas no programa por faixa etária; c) verificar o uso da mamadeira noturna pelas crianças atendidas no programa por faixa etária e d) identificar a utilização da higienização bucal ao iniciar o programa por faixa etária; e) evidenciar a prevalência da cárie dental nas crianças ao iniciarem o programa de atendimento odontológico por faixa etária.

\section{Métodos}

O presente trabalho caracteriza-se como um estudo descritivo de corte transversal.

Foi realizado levantamento junto ao arquivo do Programa de Atendimento Odontológico Precoce da Clínica Odontológica do Verdão do Município de Cuiabá, Mato Grosso, Brasil, sendo analisados todos os 934 prontuários das crianças atendidas no período compreendido entre novembro de 1996 (início do programa de atendimento odontológico precoce) a dezembro de 1998.

As informações foram coletadas a partir da ficha clínica individual de cada criança, onde foram selecionados para análise os seguintes dados: a) idade ao iniciar o tratamento; b) uso de mamadeira (sim ou não); c) uso de mamadeira durante a noite (sim ou não); d) higienização (presente ou ausente); e) cárie dentária (presente, ausente ou ausência de informação).

Deve-se ressaltar que o presente trabalho utilizou-se de dados secundários. As informações supracitadas constavam na ficha clínica do prontuário de cada criança atendida, sendo que as mesmas eram preenchidas após entrevista com a mãe e exame clínico da boca da criança. É importante salientar que dados secundários devem ser utilizados com cautela, visto que a maioria dos registros em arquivos foi produzida com um objetivo específico e para um público específico, e essas condições devem ser avaliadas por completo, a fim de interpretar a utilidade de quaisquer registros arquivados. 10

Os dados foram coletados no arquivo do programa na "Clínica Odontológica do Verdão" entre fevereiro e março de 1999.

Foi realizada uma análise estatística através de freqüência simples, relativa e acumulada para os dados coletados 


\section{Resultados}

A compilação dos dados coletados permitiu a elaboração de tabelas com as variáveis previamente colhidas e a análise das mesmas possibilitou que alguns aspectos do atendimento da "Bebê-Clínica" ficassem evidenciados.

\section{Tabela 1}

Distribuição das crianças atendidas na "Bebê-Clínica da Clínica Odontológica do Verdão", segundo a faixa etária ao iniciar-se no programa entre $1996 \mathrm{e}$ 1998. Cuiabá, Mato Grosso.

\begin{tabular}{lrrr}
\hline $\begin{array}{l}\text { Faixa etária } \\
\text { (em meses) }\end{array}$ & $\mathbf{n}$ & $\%$ & $\%$ acumulado \\
\hline $0-6$ & 149 & 15,95 & 15,95 \\
$7-12$ & 164 & 17,56 & 33,51 \\
$13-18$ & 139 & 14,88 & 48,39 \\
$19-24$ & 158 & 16,92 & 65,31 \\
$25-30$ & 117 & 12,53 & 77,84 \\
$31-36$ & 141 & 15,09 & 92,93 \\
$37-42$ & 45 & 4,82 & 97,75 \\
43 ou mais & 21 & 2,25 & 100,00 \\
Total & 934 & 100,00 & 100,00 \\
\hline
\end{tabular}

A distribuição das crianças atendidas na "BebêClínica da Clínica Odontológica do Verdão", segundo a faixa etária ao iniciar-se no programa, é mostrada na Tabela 1. A clientela do programa citado era composta, em sua grande maioria, $(92,93 \%)$ de crianças que ao iniciar o tratamento têm idade entre 0 e 36 meses; e essas crianças encontravam-se distribuídas de maneira bastante uniforme nos intervalos de seis em seis meses até completar 36 meses, restando apenas uma diminuta parcela, $7,07 \%$, de crianças que iniciaram o tratamento com 37 meses de idade ou mais.

Acerca do uso de mamadeira pelas crianças atendidas no programa, mostrado na Tabela 2 , verificamos que até os 42 meses de idade, em todas as faixas etárias o número de crianças que usava mamadeira era superior ao número de crianças que não faziam uso da mesma. Essa condição tinha início na faixa etária de 0 a 6 meses com $53,38 \%$ das crianças usando a mamadeira, e aumentava, conforme se elevava a faixa etária, chegando ao seu ápice na faixa dos 25 a 30 meses com $68,97 \%$ das crianças fazendo uso da mamadeira. Depois o uso desse dispositivo de alimentação artificial segue uma curva descendente terminando nas crianças de 43 meses ou mais onde $46,62 \%$ ainda faziam uso da mamadeira.

A distribuição das crianças segundo o uso da mamadeira noturna, dentre aquelas crianças que faziam uso da mamadeira, é mostrada na Tabela 3. É observado que, das crianças que iniciaram o tratamento na "Bebê-Clínica da Clínica Odontológica do Verdão" fazendo uso da mamadeira, a maioria absoluta o fazia também durante a noite. Isso ocorre em todas as faixas etárias: de 0 a 6 meses com 59,49\% das crianças que usavam mamadeira fazendo este uso noturno a 90,28 das crianças de 31 a 36 meses que o faziam, inclusive durante a noite.

A presença de higiene bucal, independente da forma como era realizada entre as crianças atendidas no programa é mostrada na Tabela 4. É evidenciado um aumento gradativo da realização de higiene da cavidade bucal da criança conforme sua faixa etária vai aumentando. Em 32,43\% das crianças de 0 a 6 meses que iniciaram o tratamento na "Bebê-Clínica da Clínica Odontológica do Verdão" estava presente a higiene bucal, e a prevalência da mesma aumenta conforme a idade, chegando em seu ápice na faixa etária de 25 a 30 meses com 97,41\% das crianças que procuraram o programa já fazendo a higiene bucal.

A prevalência da cárie dentária e a presença ou não dessa informação na clientela atendida no programa estudado são apresentados na Tabela 5 . Essa tabela deixa claro que a prevalência da cárie dentária nas crianças que foram atendidas na "BebêClínica da Clínica Odontológica do Verdão" é diretamente proporcional à faixa etária; enquanto $0,00 \% \mathrm{e}$ $7,32 \%$ das crianças de 0 a 6 e 7 a 12 meses de idade respectivamente apresentavam lesões de cárie; $57,45 \%, 53,33 \%$ e $87,50 \%$ das crianças que iniciaram-se no programa nas faixas etárias de 31 a 36,37 a 42 e maiores de 43 meses respectivamente já apresentavam sinais da doença.

O número de fichas onde não foi preenchido o quesito presença de cárie é muito grande, chegando a 25 fichas $(15,82 \%)$ de crianças na faixa etária de 19 a 24 meses e $27(19,15 \%)$ de crianças entre 31 e 36 meses. 
Tabela 2

Distribuição, por faixa etária, das crianças atendidas na "Bebê-Clínica da Clínica Odontológica do Verdão", segundo o uso ou não da mamadeira ao iniciar-se no programa entre 1996 e 1998. Cuiabá, Mato Grosso.

\begin{tabular}{|c|c|c|c|c|c|c|}
\hline \multirow{2}{*}{ Faixa etária (em meses) } & \multicolumn{2}{|c|}{ Com mamadeira } & \multicolumn{2}{|c|}{ Sem mamadeira } & \multicolumn{2}{|c|}{ Total* } \\
\hline & $\mathrm{n}$ & $\%$ & $\mathrm{n}$ & $\%$ & $\mathrm{n}$ & $\%$ \\
\hline $0-6$ & 79 & 53,38 & 69 & 31,90 & 148 & 100,00 \\
\hline $7-12$ & 111 & 68,10 & 52 & 31,90 & 163 & 100,00 \\
\hline $13-18$ & 94 & 67,63 & 45 & 32,37 & 139 & 100,00 \\
\hline $19-24$ & 104 & 65,82 & 54 & 34,18 & 158 & 100,00 \\
\hline $25-30$ & 80 & 68,97 & 36 & 31,03 & 116 & 100,00 \\
\hline $31-36$ & 72 & 51,43 & 68 & 48,57 & 140 & 100,00 \\
\hline $37-42$ & 23 & 51,11 & 22 & 48,89 & 45 & 100,00 \\
\hline 43 ou mais & 10 & 47,62 & 11 & 52,38 & 21 & 100,00 \\
\hline
\end{tabular}

* Foram excluídas quatro crianças cujas fichas não continham a informação uso de mamadeira.

Tabela 3

Distribuição, por faixa etária, das crianças atendidas na "Bebê-Clínica da Clínica Odontológica do Verdão" que usaram mamadeira segundo o uso durante a noite ao iniciar-se no programa entre 1996 e 1998. Cuiabá, Mato Grosso.

\begin{tabular}{|c|c|c|c|c|c|c|}
\hline \multirow{2}{*}{ Faixa etária (em meses) } & \multicolumn{2}{|c|}{ Com mamadeira noturna } & \multicolumn{2}{|c|}{ Sem mamadeira noturna } & \multicolumn{2}{|c|}{ Total* } \\
\hline & $\mathrm{n}$ & $\%$ & $\mathrm{n}$ & $\%$ & $\mathrm{n}$ & $\%$ \\
\hline $0-6$ & 47 & 59,49 & 32 & 40,51 & 79 & 100,00 \\
\hline $7-12$ & 87 & 78,38 & 24 & 21,62 & 111 & 100,00 \\
\hline $13-18$ & 78 & 82,98 & 16 & 17,02 & 94 & 100,00 \\
\hline $19-24$ & 89 & 85,58 & 15 & 14,42 & 104 & 100,00 \\
\hline $25-30$ & 68 & 85,00 & 12 & 15,00 & 80 & 100,00 \\
\hline $31-36$ & 65 & 90,28 & 7 & 9,72 & 72 & 100,00 \\
\hline $37-42$ & 17 & 73,51 & 6 & 26,09 & 23 & 100,00 \\
\hline 43 ou mais & 9 & 90,00 & 1 & 10,00 & 10 & 100,00 \\
\hline
\end{tabular}

* Foram excluídas crianças cujas fichas não continham a informação uso de mamadeira noturna.

\section{Tabela 4}

Distribuição, por faixa etária, das crianças atendidas na "Bebê-Clínica da Clínica Odontológica do Verdão", segundo a presença ou não de alguma forma de higiene bucal ao iniciar-se no programa entre 1996 e 1998. Cuiabá, Mato Grosso.

\begin{tabular}{|c|c|c|c|c|c|c|}
\hline \multirow{2}{*}{ Faixa etária (em meses) } & \multicolumn{2}{|c|}{ Com higiene bucal } & \multicolumn{2}{|c|}{ Sem higiene bucal } & \multicolumn{2}{|c|}{ Total* } \\
\hline & $\mathrm{n}$ & $\%$ & $\mathrm{n}$ & $\%$ & $\mathrm{n}$ & $\%$ \\
\hline $0-6$ & 48 & 32,43 & 100 & 67,57 & 148 & 100,00 \\
\hline $7-1$ & 56 & 34,36 & 107 & 65,64 & 163 & 100,00 \\
\hline $13-18$ & 94 & 67,62 & 45 & 32,38 & 139 & 100,00 \\
\hline $19-24$ & 130 & 82,28 & 28 & 17,72 & 158 & 100,00 \\
\hline $25-30$ & 113 & 97,41 & 3 & 2,59 & 116 & 100,00 \\
\hline $31-36$ & 131 & 94,24 & 8 & 9,76 & 139 & 100,00 \\
\hline $37-42$ & 41 & 91,11 & 4 & 8,89 & 45 & 100,00 \\
\hline 43 ou mais & 20 & 95,24 & 1 & 4,76 & 21 & 100,00 \\
\hline
\end{tabular}

* Foram excluídas cinco crianças cujas fichas não continham a informação presença de higiene bucal. 
Distribuição, por faixa etária, das crianças atendidas na "Bebê-Clínica da Clínica Odontológica do Verdão", segundo a presença ou não de alguma forma de higiene bucal ao iniciar-se no programa entre 1996 e 1998. Cuiabá, Mato Grosso.

\begin{tabular}{|c|c|c|c|c|c|c|c|c|}
\hline \multirow{2}{*}{ Faixa etária (em meses) } & \multicolumn{2}{|c|}{ Com cárie } & \multicolumn{2}{|c|}{ Sem cárie } & \multicolumn{2}{|c|}{ Sem informação } & \multicolumn{2}{|c|}{ Total } \\
\hline & $n$ & $\%$ & $\mathrm{n}$ & $\%$ & $\mathrm{n}$ & $\%$ & $n$ & $\%$ \\
\hline $0-6$ & 0 & 0,00 & 140 & 93,96 & 9 & 6,04 & 149 & 100,00 \\
\hline $7-12$ & 12 & 7,32 & 129 & 78,66 & 23 & 14,02 & 164 & 100,00 \\
\hline $13-18$ & 28 & 20,14 & 93 & 66,91 & 18 & 12,95 & 139 & 100,00 \\
\hline $19-24$ & 64 & 40,51 & 69 & 43,67 & 25 & 15,82 & 158 & 100,00 \\
\hline $25-30$ & 57 & 48,72 & 38 & 32,48 & 22 & 18,80 & 117 & 100,00 \\
\hline $31-36$ & 81 & 57,45 & 33 & 23,40 & 27 & 19,15 & 141 & 100,00 \\
\hline $37-42$ & 24 & 53,33 & 12 & 26,67 & 9 & 20,00 & 45 & 100,00 \\
\hline 43 ou mais & 21 & 87,50 & 1 & 4,17 & 2 & 8,3 & 24 & 100,00 \\
\hline
\end{tabular}

\section{Discussão}

O grande número de crianças que iniciaram no Programa com idade superior a 12 meses, demonstra que o Programa de Atendimento Odontológico Precoce sofreu algumas alterações ao ser implantado pela rede municipal de saúde de Cuiabá. Apesar do primeiro ano de vida (0 a 12 meses) ser considerado o momento ideal para o início do tratamento odontológico, 4,7,11,12 dentre a clientela estudada, observamos que dois terços dos pacientes $(66,49 \%)$ iniciaram o programa com idade superior à preconizada.

É importante salientar que os benefícios relacionados ao aleitamento natural estão bem estabelecidos, sendo considerado o mais desejável método de alimentação infantil, no que diz respeito aos aspectos físiológicos, físicos e psicológicos. O leite materno é um alimento especialmente adaptado para atender às necessidades nutricionais do bebê, providenciando energia para seu desenvolvimento e crescimento e fornecendo proteção contra infecções e alergias. Quanto ao papel no desenvolvimento psicológico do bebê, através do contato corporal com a mãe, durante a amamentação, a criança desenvolve seus sentimentos de bem-estar, segurança e afetividade. 13

Além disso, a amamentação no seio materno representa para a criança fator inicial de um bom desenvolvimento dento-facial, permitindo a obtenção de uma oclusão dentária normal e conseqüentemente instalação de uma mastigação futura correta, segundo Moyers citado por Czernay et al. 14

Considerando os benefícios que o aleitamento materno traz para a criança e sua mãe, os dados apresentados são importantes tanto no contexto da saúde bucal como na saúde geral; os dados mostram que as crianças que freqüentam a "Bebê-Clínica da Clínica Odontológica do Verdão" estão sendo desmamadas precocemente, já que é orientado o aleitamento natural exclusivo e na livre demanda até os seis meses de vida. 4

A mamadeira não apresenta restrições sociais, tem boa aceitação pelas crianças, já que normalmente seu conteúdo é adoçado, é de fácil manipulação pela criança e pode ser utilizada a qualquer hora e ser fornecida pela mãe ou outra pessoa. Esses aspectos do hábito de utilização da mamadeira contribuem para a caracterização de um padrão de alta freqüência e prolongados períodos de uso. 15

Atualmente, tem-se sugerido a substituição da mamadeira por outros métodos de aleitamento artificial, quando por alguma razão a criança estiver impossibilitada de ser amamentada. Nesses casos, o uso exclusivo de copos ou xícaras desde o nascimento vem sendo preconizado. 14

Além dos benefícios do aleitamento natural, bem como dos malefícios ocasionados pelo aleitamento artificial, o uso da mamadeira pode causar diversos prejuízos para a criança: confusão de bicos com o seio da mãe, fazendo a criança largar o aleitamento natural precocemente; falta do estímulo correto da musculatura e osso da face; desenvolvimento de deglutição atípica, entre outros.2,4,16,17 Além disso, com o uso da mamadeira durante a noite, resíduos de leite (geralmente misturados com suplementos tais como açúcar, achocolatados e farinhas ricas em carboidratos) permanecerão por maior tempo na 
cavidade bucal em contato direto com os dentes devido à diminuição fisiológica do fluxo salivar e do reflexo de deglutição que ocorrem durante a noite e, com isso, aumentando o risco da criança vir a desenvolver a doença cárie. 18,19

Com os novos conhecimentos sobre etiologia e patologia da cárie dentária, muitos conceitos foram modificados na prática odontológica, dentre eles o tratamento preventivo da doença. ${ }^{20}$ Sabe-se que a incidência da cárie dentária está intimamente relacionada com a dieta e higienização dos dentes.2,4,16,21 A American Dental Association (ADA) já em 1981,orientou que a higienização da boca tivesse início antes do irrompimento dos dentes, para tornar o campo mais limpo, assim como acostumar a criança à manipulação de sua boca. 4 Walter et al. 4 preconizam o início da limpeza com o irrompimento dos primeiros dentes, principalmente à noite após a última mamada.

É muito importante que os responsáveis pelos bebês, principalmente as mães, tenham consciência que quanto mais cedo eles começarem a fazer a limpeza da boca da criança, menor chance ela terá de apresentar lesões de cárie.

Vale ressaltar que a realização da higiene da boca da criança, principalmente a desdentada, não precisa ser feita com escova dental, podendo ser realizada com uma gaze ou uma fralda bem limpa separada para ser utilizada somente com esse fim. 4,20

A relação entre a prevalência da cárie dentária e a faixa etária das crianças atendidas na "Bebê-Clínica da Clínica Odontológica do Verdão" segue a tendência de outros levantamentos. $22-24$ Pode-se dizer que essa diferença está associada em parte à erupção dentária as crianças menores de seis meses em sua grande maioria são desdentadas, não havendo a possibilidade de manifestar sinais da doença cárie; por outro lado, os últimos dentes decíduos a irromper fazem-no por volta dos 36 meses, completando a dentição decídua e nesse momento $57,45 \%$ das crianças já apresentam dentes cariados.

A atenção precoce é uma conquista da Odontologia, e representa a incorporação de um novo entendimento da abordagem das doenças bucais fortemente centrada numa perspectiva preventivo promocional. Surgida a partir do desenvolvimento da Cariologia, a sua incorporação na prática deveu- se à disseminação da idéia do tratamento da cárie enquanto doença infecciosa, reforçando a importância do controle dessa o mais precocemente possível. 25

Guimarães et al.,25 citando Morinushi, relatam que o aparecimento de cárie em crianças de baixa idade está diretamente relacionado à negligência materna. Os autores relatam ainda que a maior parte das mães ainda é carente de informações a respeito desses problemas. Muitas desconhecem as causas que levam ao comprometimento dos dentes dos seus filhos na primeira infância, os hábitos de higiene que devem ser introduzidos desde cedo, a importância de o bebê receber assistência odontológica antes do primeiro ano de vida, e que o fator dieta nessa idade também está diretamente relacionado com a cárie.

É muito importante ficar claro que a cárie dentária é uma doença, não uma condição fisiológica, e não faz parte da infância, assim como a dentadura (prótese total) não faz parte da senilidade.

\section{Conclusões}

A incorporação da Odontologia para Bebês pelo modelo de assistência odontológica do Município de Cuiabá amparou as crianças que não eram beneficiadas pelos programas de saúde escolares.

A clientela da "Bebê-Clínica da Clínica Odontológica do Verdão" apresentou um alto índice de desmame precoce, onde $53,38 \%$ das crianças com idade entre 0 e 6 meses faziam uso de mamadeira.

Em todas as faixas etárias estudadas, com exceção das crianças de 43 meses ou mais, mais de $51 \%$ das crianças fazem uso de mamadeira.

Dentre as crianças que fazem uso de mamadeira, entre $59,49 \%$ ( 0 - 6 meses) e 90,28\% (31 - 36 meses) mamam durante a noite.

A higiene bucal esteve presente no primeiro ano de vida em apenas um terço $(32,43 \%$ de 0 - 6 meses e $34,36 \%$ de 7 - 12 meses) das crianças assistidas pela "Bebê-Clínica da Clínica Odontológica do Verdão".

A prevalência da cárie dental está diretamente associada com a idade das crianças assistidas pela "Bebê-Clínica da Clínica Odontológica do Verdão".

É necessária a realização de um trabalho de conscientização da população com relação à saúde bucal das crianças em idade pré-escolar. 


\section{Referências}

1. VIII Conferência Nacional de Saúde; 1986 mar 17-21; Brasília, DF. Reformulação do Sistema Nacional de Saúde: relatório final. Brasília (DF): Ministério da Saúde; 1986.

2. Corrêa MSNP. Odontopediatria na primeira infância. São Paulo: Santos; 1998

3. Berlinger G. A doença. São Paulo: Hucitec; 1988

4. Walter LRF, Ferelle A, Issao M. Odontologia para o bebê. Odontopediatria do nascimento aos três anos. São Paulo: Artes Médicas; 1997.

5. Oliveira AGRC, Arcieri RM, Unfer B, Costa ICC, Moraes E, Saliba NA. Modelos assistenciais em saúde bucal no Brasil: tendências e perspectivas. Ação Coletiva 1999; 2: 9-14.

6. Pinto VG. Saúde bucal coletiva. 4. ed. São Paulo: Santos; 2000 .

7. Baldani MHP, Lopes CMDL, Kriger L, Matsuo T. A odontologia para bebês no Estado do Paraná, Brasil: perfil do Programa de Atenção Precoce à Saúde Bucal. J Bras Odontopediatr Odontol Bebê 2003; 6: 210-6.

8. Nakagawa OY. Projeto Plano de Atendimento Odontológico no Primeiro Ano de Vida. Bebê-Clínica no município de Cuiabá. Cuiabá: Fundação de Saúde de Cuiabá; 1995.

9. Maciel SSSV. Prevalência de cárie dentária nas crianças de 7 a 48 meses de idade, da Clínica Odontológica do Verdão, Distrito Sanitário Oeste, Cuiabá, MT [especialização]. Cuiabá: Instituto de Saúde Coletiva da Universidade Federal de Mato Grosso; 1998.

10. Yin RK. Estudo de caso: planejamento e métodos. 2. ed. Porto Alegre: Bookman; 2001.

11. Carneiro AA, Miasato JM, Gama RS, Batti P. Estudo comparativo dos motivos das consultas do Projeto Bebê Clínica UNIGRANRIO. In: Anais do III Encontro Nacional de Odontologia para Bebês; 2000 jul 3-5; Araçatuba, SP. São Paulo: FOA/UNESP; 2000. p. 21. [resumo 24].

12. Thomas HF. First dental visit, first birthday: a rationale and protocol for infant oral health care. Tex Dental J 1997; 114: 15-9.

13. Tollara MN, Corrêa MSNP, Bönecker MJS, Carvalho GD Aleitamento natural. In: Corrêa MSNP. Odontopediatria na primeira infância. São Paulo: Santos; 1998. p. 71-86.
14. Czernay APC, Nogueira DA, Shardosim LR, Bosco VL. Pode o copo substituir a mamadeira como método alternativo de aleitamento artificial para bebês? J Bras Odontopediatr Odontol Bebê 2003; 6: 235-9.

15. Fraiz FC. Dieta e cárie na primeira infância. In: Walter LRF, Ferelle A, Issao M. Odontologia para o bebê. Odontopediatria do nascimento aos 3 anos. São Paulo: Artes Médicas; 1997.

16. Guedes-Pinto AC. Odontopediatria. 5. ed. São Paulo: Santos; 1995.

17. King FS. Como ajudar as mães a amamentar. Londrina: AMREF; 1997.

18. Dureis IT, Moreira SC. Risco de cárie em bebês. Rev Odontol Brasil Central 1995; 14: 11-7.

19. Holst A, Martensson I, Laurin M. Identification of caries risk children and prevention of caries in pre-school children. Swed Dent J 1997; 21: 185-91.

20. Moura LFAD, Rebelo MCCBL, Moura MD, Arêa Leão VL. Avaliação da eficácia de métodos de higiene bucal em bebês. J Bras Odontopediatr Odontol Bebê 2000; 3: 141-6.

21. Meneghim MC. Dieta, nutrição e cárie dentária. In: Pereira AC, organizador. Odontologia em saúde coletiva: planejando ações e promovendo saúde. Porto Alegre: Artmed; 2003. p. 301-17.

22. Montandon EM, Alves TDB, Menezes VA. Levantamento epidemiológico em crianças de 0 a 30 meses na cidade do Recife, PE. Parte II: Prevalência de patologias bucais. Rev Odontol Brasil Central 1998; 7: 32-6.

23. Garboza CS, Walter LRF. Estudo da prevalência de cárie numa população de 0 a 5 anos atendida precocemente pela Bebê-Clínica da Universidade Estadual de Londrina. Semina 1997; 18: 51-4.

24. Morita MC, Walter LRF, Guillain M. Prevalência de cárie dentária em crianças brasileiras com idade de 0 a 36 meses. Rev Odontol Brasil Central 1992; 2: 17-9.

25. Guimarães AO, Costa ICC, Oliveira ALS. As origens, objetivos e razões de ser da odontologia para bebês. J Bras Odontopediatr Odontol Bebê 2003; 6: 83-6.

Recebido em 9 de janeiro de 2004

Versão final apresentada em 7 dezembro de 2004

Aprovado em 10 de janeiro de 2005 